\title{
Microarray-based genomic profiling and in situ hybridization on fibrotic bone marrow biopsies for the identification of numerical chromosomal abnormalities in myelodysplastic syndrome
}

Marian JPL Stevens-Kroef ${ }^{1 *}$, Konnie M Hebeda², Eugène T Verwiel ${ }^{1}$, Eveline J Kamping ${ }^{1}$, Patricia H van Cleef ${ }^{2}$ Roland P Kuiper ${ }^{1}$ and Patricia JTA Groenen ${ }^{2}$

\begin{abstract}
Background: Myelodysplastic syndromes (MDS) are a heterogeneous group of clonal hematological malignancies. In MDS patients with a fibrotic bone marrow the aspiration of cells often fails (dry-tap), which hampers standard karyotyping. Obtaining genetic data from these fibrotic marrows is therefore challenging, and up till now in situ hybridization applied to bone marrow biopsies is the only option. The microarray-based genomic profiling technology has already proven its value for bone marrow aspirates and peripheral blood samples, but has never been applied to the technically challenging bone marrow biopsies. We describe an approach for microarray-based genomic profiling on bone marrow biopsies and demonstrate its ability to obtain clinically relevant cytogenetic aberrations. In addition the data were compared with those obtained by in situ hybridization and karyotyping.

Results: We have evaluated the success rate of microarray-based genomic profiling by studying twenty-one bone marrow biopsies (7 fibrotic MDS, 12 non-fibrotic MDS and 2 reactive), by microarray-based genomic profiling and in situ hybridization (12 of 21 cases). The data obtained with these techniques were compared with conventional karyotyping data on corresponding bone marrow aspirates. Of the 15 copy number aberrations that were detected by in situ hybridization, 13 were concordant with microarray-based genomic profiling and karyotyping, whereas two hybridizations were misinterpreted. In 20 of 21 patients, the data obtained by microarray-based genomic profiling and karyotyping were identical or differences could be explained by the presence of marker chromosomes, complex karyotypes, clonal heterogeneity or disease progression.

Conclusions: We demonstrate that genome wide microarray-based genomic profiling performed on bone marrow biopsies has a similar success rate compared to in situ hybridization, and prevents misinterpretation of chromosomal losses as observed by FISH. In addition, equal to even higher resolutions were obtained with genomic profiling compared to conventional karyotyping. Our findings indicate that microarray-based profiling, even on bone marrow biopsies, is a valid approach for the identification of genetic abnormalities. This is a valuable substitution in cases of fibrotic MDS lacking cytogenetic results.
\end{abstract}

Keywords: Microarray, in situ hybridization, Karyotyping, MDS, Fibrotic, Bone marrow biopsy

\footnotetext{
* Correspondence: Marian.Stevens-Kroef@radboudumc.nl

${ }^{1}$ Department of Human Genetics, Radboud university medical center,

P.O. Box 9101, 6500 HB Nijmegen, The Netherlands

Full list of author information is available at the end of the article
} 


\section{Background}

Myelodysplastic syndrome (MDS) is a heterogeneous group of hematopoietic neoplasms characterized by bone marrow dysplasia and ineffective hematopoiesis which causes peripheral cytopenias and a risk of progression to acute myeloid leukemia (AML). The diagnosis and classification of MDS is based largely on clinicopathological, morphological and cytogenetic findings. In addition, chromosomal abnormalities are found in $40-60 \%$ of the patients and prove helpful for determination of prognostic status with regard to survival and AML evolution [1,2]. For instance in the International Prognostic Scoring System (IPSS) for MDS, cytogenetic subgroups of outcome were defined as follows: "good" outcomes were associated with a normal karyotype, $-\mathrm{Y}$ alone, del (5q) alone, del (20q) alone; "poor" outcomes showed complex karyotypes (ie, $\geq 3$ abnormalities) or chromosome 7 anomalies; and "intermediate" outcomes were associated with all other abnormalities [1]. Conventional karyotyping is the current standard screening for chromosomal abnormalities on metaphases. This approach can be problematic in cases with fibrotic bone marrow biopsies, often resulting in none or a non-representative bone marrow aspirate sample (dry-tap) which does not contain neoplastic blasts. Such a dry-tap is observed in $4-7 \%$ of the bone marrow examinations [3,4]. In situ hybridization (ISH) using targeted probes to detect recurrent chromosomal trisomies and translocations has been applied to bone marrow biopsies in only a limited number of studies in MDS [5-8]. The reliable detection of losses of chromosomes, and especially loss of chromosome segments is difficult in tissue sections, since nuclei are generally only partly present on the slide due to cutting of the tissue, which may result in false positive identification of chromosomal losses [5]. In addition, there can be a significant intermingling of neoplastic and non-neoplastic cells in MDS biopsies, resulting in difficulties in the identification of genetically abnormal neoplastic cells. The combination of fluorescence in situ hybridization (FISH) and the detection of immunophenotypic markers in formalin fixed paraffin-embedded (FFPE) sections of bone marrow can overcome the problem of a low percentage of malignant cells [7].

Genome wide profiling using bacterial artificial chromosome (BAC) libraries, oligonucleotide and single nucleotide polymorphism-based microarray platforms has been applied in a research or exploratory setting to profile the genomic alterations in patients with MDS and AML [9-13]. However, these microarray-based genome profiling approaches have only been applied to highmolecular weight DNA isolated from bone marrow aspirates or peripheral blood samples. The experience with DNA obtained from formalin-fixed paraffin embedded
(FFPE) bone marrow biopsies is very limited, but this source would be helpful in MDS patients with bone marrow fibrosis resulting in a non-representative bone marrow aspirate and subsequent lack of cytogenetic data. The major disadvantage of DNA from tissue blocks is the fragmentation and degradation during the formaldehyde fixation and decalcification process.

To evaluate the diagnostic value of microarray-based genomic profiling, especially on fibrotic bone marrow samples, we have compared microarray-based genomic profiling and FISH or Chromogenic in situ hybridization on bone marrow biopsies with data obtained by karyotyping of corresponding bone marrow aspirate samples in MDS patients with and without extensive fibrosis in the bone marrow biopsy. Despite its limitation in detecting (recurrent) balanced translocations and the lower sensitivity, we demonstrate that, also in patients with a fibrotic bone marrow, microarray-based genomic profiling using DNA obtained from bone marrow FFPE-biopsies has a similar success rate as compared to in situ hybridization applied to bone marrow biopsies. In addition, microarray-based genomic profiling overcomes the false positive identification of chromosomal losses that occurred in FISH, can identify genomic abnormalities which are outside the scope of the ISH probes applied, and has a comparable or even higher resolution than karyotyping, since copy number abnormalities $>10 \mathrm{Mb}$ in size can be easily detected.

\section{Results}

Interpretation criteria of microarray data and description of genomic profiles

Tissues from FFPE trephine biopsy specimens of 24 MDS patients and 2 reactive non-fibrotic cases, all with a successful cytogenetic result from a simultaneous bone marrow aspirate, were initially selected. The quality of the extracted genomic DNA was assessed by its ability to amplify of control gene fragments of $200 \mathrm{bp}$ or more. However, three of the 26 FFPE specimens had to be excluded due to an insufficient quality and/or quantity of DNA. A microarray-based genomic profile was obtained from genomic DNA from the other 23 patients. Two additional samples had to be excluded because of the noise level of the obtained array profiles as measured by the quality score in Nexus. Therefore, successful genomic profiling could be performed in 21 of 23 profiled cases (91\%). We have divided the patients in three groups (Table 1). Group 1 consisted of 7 non-fibrotic MDS cases with cytogenetic abnormalities. Group 2 were 7 MDS cases with availability of an abnormal karyotype despite bone marrow fibrosis. Group 3 included 4 nonfibrotic MDS cases, 1 AML case and 2 biopsies of reactive bone marrow, all with a normal karyotype except for a balanced translocation of chromosome 3 in one case. 
Table 1 In situ hybridization, microarray and cytogenetic data of the patients

\begin{tabular}{|c|c|c|c|c|c|c|c|c|}
\hline Group & Patient ID & Sex & Age (years) & Diagnosis & Fibrosis & FISH (BM biopsy) & Array based karyotype (BM biopsy) & Karyotype (BM aspirate) \\
\hline \multirow[t]{7}{*}{1} & 4902 & M & 63 & RCMD & no & not done & $\operatorname{del}(16)(q 11 q 23)$ & $46, X Y$, del (16)(q11q23)[4]/46,XY[6] \\
\hline & 4519 & $\mathrm{~F}$ & 59 & AML & no & loss 7 (44\%) & $\begin{array}{l}\text { del(3)(p24p22),del(3)(p14p12),del(4) } \\
\text { (p15),dup(5)(p11),del(5)(q11 q35),del } \\
\text { (7)(p14p11), del (7)(q11q26),del(10) } \\
\text { (q11q24),del(10)(q25q26),dup(22)(q11) }\end{array}$ & $45, X X$, del $(5)(q 13 q 33),-7[4] / 46, X X[16]$ \\
\hline & 4898 & $\mathrm{~F}$ & 42 & $\mathrm{RCMD}$ & no & loss 7 (50\%) & $-7,+21,+22$ & $45, X X,-7[4] / 47, s 1,+21,+22[5] / 46, X X[1]$ \\
\hline & 4893 & M & 80 & RAEB-1 & no & trisomy 8 (50\%) & +8 & $47, X Y,+8[10]$ \\
\hline & 4895 & $\mathrm{~F}$ & 59 & RCMD-RS & no & trisomy 8 (23\%) & +8 & $47, X X,+8[6] / 46, X X[4]$ \\
\hline & 4896 & M & 72 & $\mathrm{RCMD}$ & no & loss 8 (47\%) loss 7 (50\%) & del(5)(q21q31),-7 & $\begin{array}{l}\text { 44,XY,del(5)(q15q33),-7,dic(15;17) } \\
\text { (p11;p11),-18,der(21;22)(q10;q10),+2mar } \\
{[\text { cp8]/46,XY[2] }}\end{array}$ \\
\hline & 4912 & M & 64 & RAEB-2 & no & not done & $+8,+21$ & $47, \mathrm{XY},+21[1] / 48, \mathrm{Sl},+8[9]$ \\
\hline \multirow[t]{7}{*}{2} & 4522 & M & 57 & $\mathrm{RCMD}$ & grade 3 & loss 20q (32\%) trisomy 8 (18\%) & $\begin{array}{l}\text { del(3)(p24q25),del(5)(q14q34),del(6) } \\
\text { (p24q16), del(7)(q11q32),+8,del (20)(q11) }\end{array}$ & $\begin{array}{l}\text { 40 44,XY,-5,-6,-7,+8,-20,+4mar } \\
{[\text { cp } 7] / 46, X Y[3]}\end{array}$ \\
\hline & 4894 & M & 67 & $\mathrm{RCMD}$ & grade 2 & loss 20q (77\%) & $\operatorname{del}(20)(q 11 q 13)$ & $46, X Y, \operatorname{del}(20)(q 13)[10]$ \\
\hline & 4899 & M & 58 & RAEB & grade 3 & not done & $\begin{array}{l}\text { del(2)(p23),del(4)(p12),del(4)(q12q13.2), } \\
\text { del(5)(q14q35),del(7)(q21q36),del(12) } \\
\text { (p12p13),del(20)(q11q13) }\end{array}$ & $\begin{array}{l}\text { 44 47,XY,-2,-2,-4,del(4)(q31),-5,-7, add } \\
\text { (9)(q34),add(12)(p13),-13,-15,-16,-17, } \\
\text { add(17)(p13),del(20)(q11),+3 7mar } \\
\text { [cp8]/46,XY[2] }\end{array}$ \\
\hline & 4903 & M & 58 & AML & patchy fibrosis & faillure & no CNA & $\begin{array}{l}\text { 44 47,XY,-2,-2,-4,-4,+6,-7,-9,-10,-13,-13,-17, } \\
\text { add(17)(p13),-19,del(20)(q11),+21,,+21, } \\
+4 \sim 8 m a r[c p 8] / 46, X Y[2]\end{array}$ \\
\hline & 4523 & $\mathrm{~F}$ & 58 & RAEB-2 & grade 3 & loss 7 (46\%) loss 5 (29\%) & $\operatorname{del}(5)(q 14 q 33),-7$ & $\begin{array}{l}45, X X, t(4 ; 17 ; 20)(q 31 ; q 12 ; q 13), \text { del(5) } \\
(q 13 q 33),-7[10]\end{array}$ \\
\hline & 4520 & M & 70 & RAEB-2 & grade 3 & loss 7 (61\%) loss 5 (40\%) & $-Y$,del(5)(q11q34),-7,del(17)(p13),-18 & $\begin{array}{l}\text { 42 44,X,-Y,-5,-7,+?12,-13,add(17) } \\
\text { (p13),-20,-21,+3mar[cp13] }\end{array}$ \\
\hline & 4521 & $\mathrm{~F}$ & 59 & $\mathrm{RCMD}$ & grade 2 & loss 5q (19\%) & $\begin{array}{l}\text { del(4)(q24q26),del(5)(q13q35),del(6) } \\
\text { (q11q21), del(7)(q11),del (17)(p13.1p13.3) }\end{array}$ & $\begin{array}{l}\text { 45 46,XX,add(1)(q32), del(5)(q?22q33), } \\
\text { del(6)(p22),add(7)(q11),-10,-13,del(17) } \\
\text { (p11),+2 3mar[cp10] }\end{array}$ \\
\hline \multirow[t]{7}{*}{3} & 4904 & $\mathrm{~F}$ & 60 & RAEB-2 & no & not done & no CNA & $46, X \times[20]$ \\
\hline & 4906 & $\mathrm{~F}$ & 64 & vit. B12 deficiency & no & not done & no CNA & $46, X X[23]$ \\
\hline & 4897 & M & 71 & hypoplastic AML & no & not done & no CNA & $46, X Y[20]$ \\
\hline & 4518 & $\mathrm{~F}$ & 45 & pure red cell aplasia & no & loss 7q (34\%) & no CNA & $46, X X[20]$ \\
\hline & 4890 & M & 58 & $\mathrm{RCMD}$ & no & not done & no CNA & $46, X Y[20]$ \\
\hline & 4892 & $\mathrm{~F}$ & 63 & RCMD-RS & no & not done & no CNA & $46, X X, t(3 ; 3)(q 21 ; q 26)[11]$ \\
\hline & 4900 & $\mathrm{~F}$ & 63 & RAEB-1 & no & not done & no CNA & $46, X X[20]$ \\
\hline
\end{tabular}

$B M=$ bone marrow. 
For comprehensive analysis and interpretation of the microarray-based genomic profiling the profiles of the 7 patients without copy number alterations (CNA) as determined by karyotyping (group 3), were evaluated to set up the interpretation criteria. In all cases that fulfilled the quality score in Nexus, small sized aberrations up to $10 \mathrm{Mb}$ in size were observed, which we have considered as normal genomic variants or background noise. Therefore the threshold for identification of a CNA was set at $10 \mathrm{Mb}$. This threshold is in line with the resolution of karyotyping. For copy number gains of chromosome 19 the threshold was set at $30 \mathrm{Mb}$, since it has been reported that the high GC content of this chromosome can result in different amplification efficiency, and that hybridization of the amplified products can result in erroneously assigned alterations of chromosome 19 on BAC library-based array-platforms [14]. Only aberrations fulfilling the above criteria were included in the genomic profiles. Since MDS samples can harbor complex patterns of cytogenetic abnormalities ( $\geq 3$ abnormalities) [1,2], the description of the obtained genomic profiles using the standardized ISCN 2013 [15] nomenclature system may result in complex reports. Therefore, we have chosen to convert the genomic array profiles into so-called microarraydeduced copy number karyotypes, adapted from the ISCN nomenclature for conventional karyotyping as has been proposed by Simons at al. [16], thus allowing a comprehensive comparison of microarray-based genomic profiling data to conventional karyotyping data.

\section{Comparison of microarray-based genomic profiling and in situ hybridization on bone marrow biopsies}

Parallel microarray-based genomic profiling and in situ hybridizations using relevant probe-sets were applied to 12 of the 21 patients, which covers 15 different probe hybridizations. For an unbiased comparison the microarray and in situ hybridization data were analyzed in a fully blinded fashion. In four patients (4893, 4895, 4894 and 4523) concordant results were obtained by in situ hybridization and microarray-based genomic profiling (Figure 1). In six cases (4519, 4898, 4896, 4522, 4520 and 4521) additional genetic abnormalities were observed by microarray, while in two cases (4896 and 4518) in situ hybridization resulted in the identification of an additional chromosome loss, not observed by microarray and karyotyping. In one other case (4903) FISH was unsuccessful (Table 1). In situ hybridization and microarray-based genomic profiling were thus concordant in 13 of the 15 (87\%) successful in situ hybridizations. These cases all involved the detection of a trisomy (cases 4893, 4895, 4522) or the losses of whole chromosomes 5 and 7 using FISH probe sets based on two probes in different colors (cases 4519, 4898, 4896, 4523, 4520).
For assessment of the detection limit of the microarray platform we have compared the genetic abnormalities as identified by microarray-based genomic profiling with those as determined by FISH on the same bone marrow biopsy. Abnormalities present in more than $18 \%$ of the cells as determined by FISH were readily detected by microarray, including 3 cases with a trisomy 8 (cases 4522 (18\%), $4895(23 \%)$ and $4893(50 \%)), 3$ patients with $\operatorname{del}(5 q)$ (cases 4521 (19\%), $4523(29 \%)$ and $4520(40 \%)) 2$ patients with del(20q) (cases 4522 (32\%) and 4894 (77\%)) and 5 patients with loss of chromosome 7 (cases 4519 (44\%), 4898 (50\%), 4896 (50\%), 4523 (46\%) and $4520(61 \%))$.

\section{Comparison of microarray-based genomic profiling and karyotyping}

In all 21 patients with successful karyotyping on the bone marrow aspirates microarray-based genomic profiling was successfully performed on the bone marrow biopsies (Table 1). No significant difference in genomic aberrations was observed between the MDS patients without (group 1) and with (group 2) fibrotic bone marrow. In 7 (pseudo) diploid patients (group 3) CNA were neither demonstrated by microarray-based genomic profiling nor by karyotyping. Conventional karyotyping on two patients revealed the presence of balanced genomic abnormalities, which as expected were not detected by microarray-based genomic profiling. One case (4892) without CNA involved the balanced $\mathrm{t}(3 ; 3)$ (q21;q26), and in the other case $(4523)$ the $t(4 ; 17 ; 20)$ was present as additional abnormality. In seven patients the CNA obtained by microarray-based genomic profiling and karyotyping were exactly identical. In five other cases with complex karyotypes (4896, 4522, 4899, 4520 and 4521) there was no exact match between microarray-based genomic profiling and karyotyping (Figure 2). However, the discrepancy could be explained by the presence of marker chromosomes which are (in part) derived from missing chromosomes. In four of these patients (4522, 4899,4520 and 4521) the loss of a chromosome or chromosome segment and the presence of marker chromosomes could be correlated with complex abnormal array profiles of the corresponding chromosomes or chromosome segments (Figure 2). In addition, all cases involved a so-called composite karyotype, indicating the presence of clonal heterogeneity. Due to clonal heterogeneity chromosome abnormalities may be present in a small fraction of the cells and remain undetected by microarray-based genomic profiling. One of the patients (case 4521) harbored a focal 12-Mb loss involving the 4 q24 region which contains the in MDS patients recurrently mutated TET2 gene [11], which was most likely missed by karyotyping due to its size below the cytogenetic resolution (Figure 2). In two patients there was 


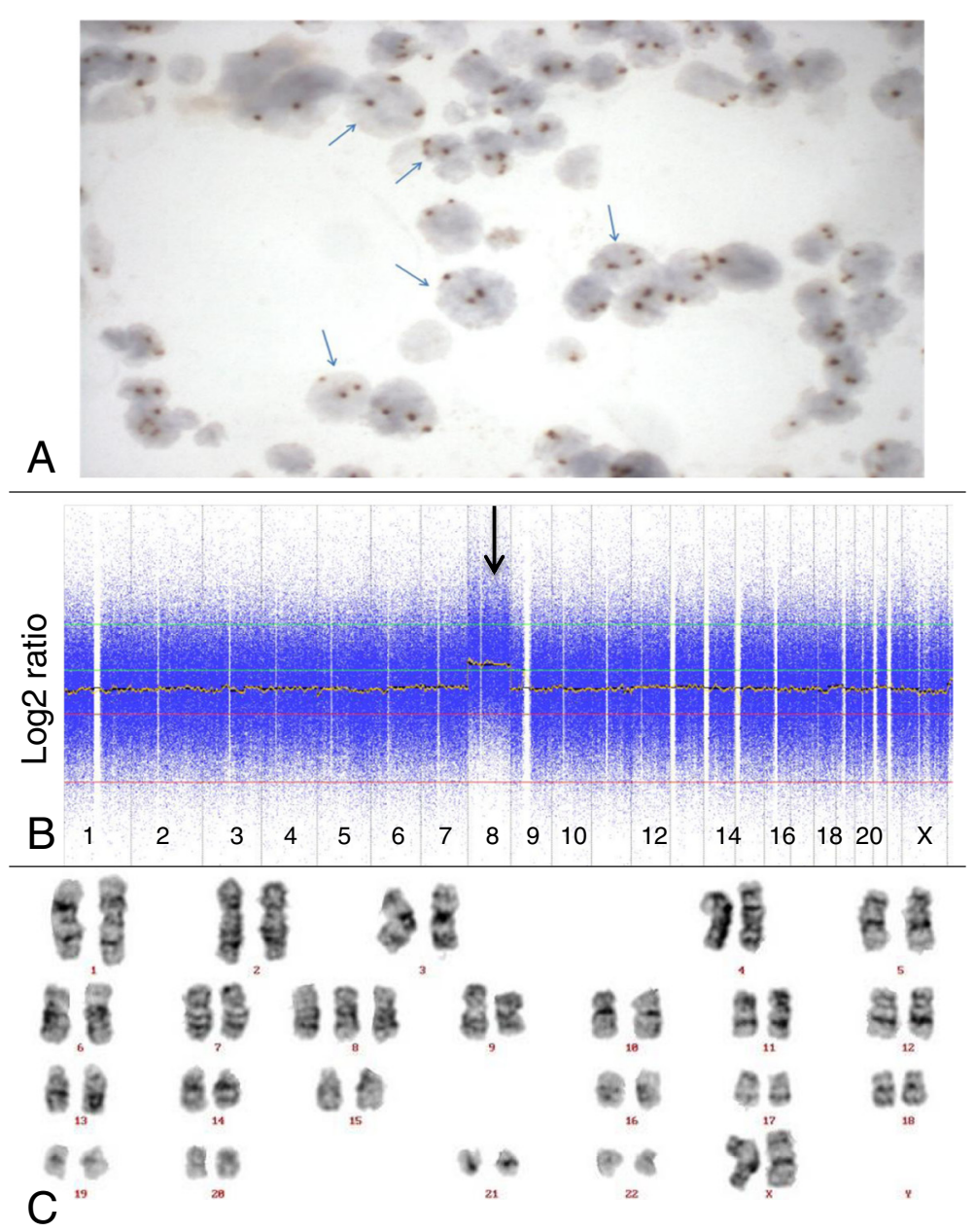

Figure 1 Trisomy 8 detected by Chromogenic in situ hybridization, karyotyping and microarray-based genomic profiling. Chromogenic in situ hybridization with probe for centromere of chromosome 8 (panel A), microarray-based genomic profile (panel B) and karyotype (panel C) of patient 4895, all indicating a trisomy 8.

a discrepancy between karyotyping and microarraybased genomic profiling. In one of these patients (case 4519) next to the del (5q) and loss of chromosome 7 as observed by karyotyping, additional genetic abnormalities were observed by microarray profiling. In this patient the microarray analysis was performed on a sample obtained 5 months later than the sample used for karyotyping, and might reflect the disease progression that was also demonstrated by histological and cytological analysis in this patient. The discrepancy in patient 4903 with a complex abnormal karyotype in the aspirate and a normal microarray-based genomic profile derived from the bone marrow biopsies may be explained by the sparse hematopoiesis present in the bone marrow biopsy of this patient, possibly resulting in a DNA-sample consisting of predominantly nonhematopoietic cells.

\section{Discussion}

Currently karyotyping is the gold standard for the detection of prognostic relevant chromosomal aberrations in MDS [1,2]. However, its application is limited to dividing cells obtained from bone marrow aspirates. In MDS patients with fibrotic bone marrow, the marrow aspirate sample is often not representative (dry tap), and thus not suitable for karyotyping [3,4]. Although challenging, analysis of bone marrow biopsies by FISH or microarraybased genomic profiling could represent an alternative approach for detection of genetic abnormalities in these MDS cases with fibrotic bone marrow.

Numerous studies have demonstrated that FISH and microarray-based genomic profiling on cells obtained from bone marrow aspirates can identify genetic abnormalities [13,17-19]. However, despite the importance of the use of bone marrow biopsies for the diagnosis of 


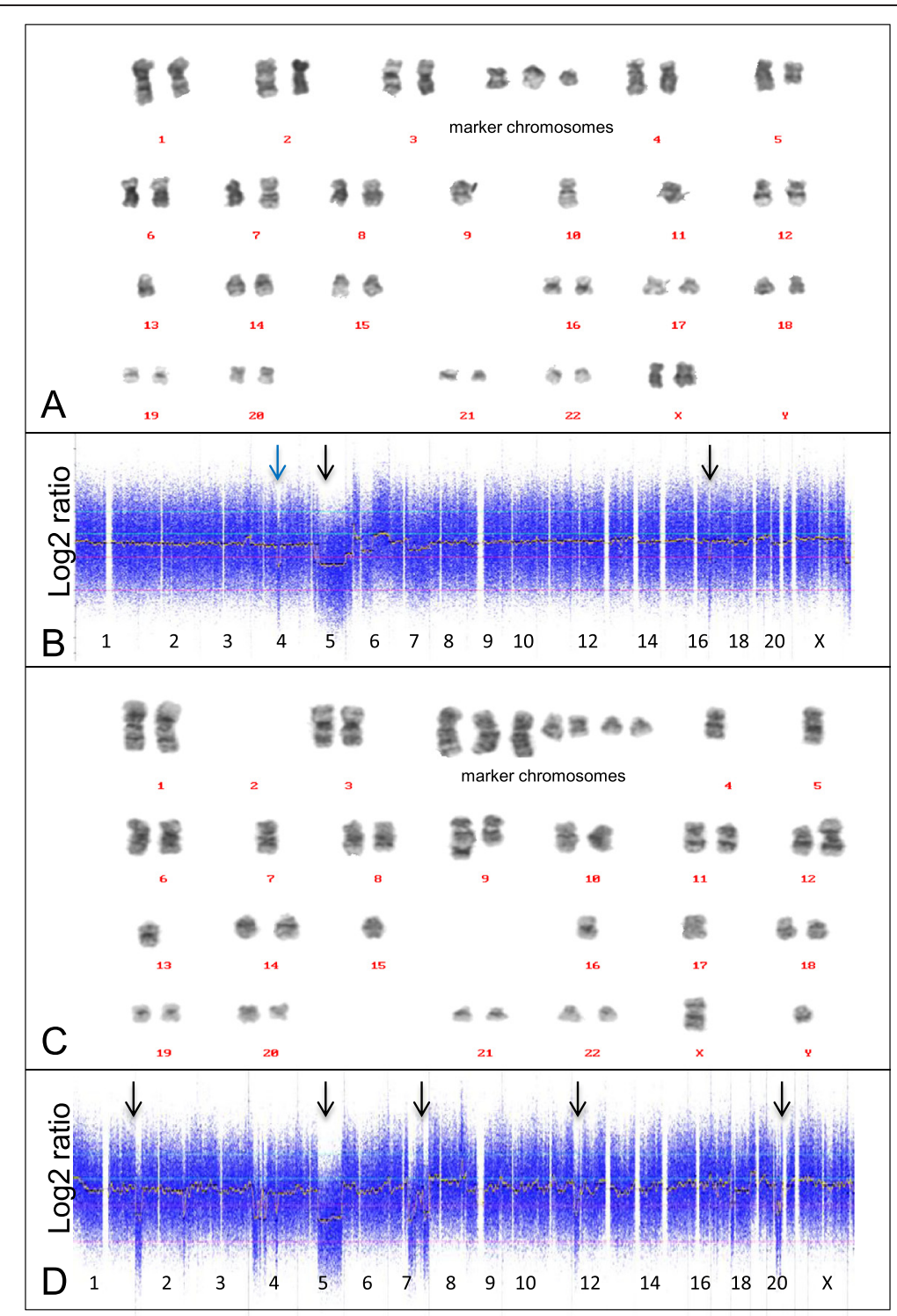

Figure 2 Comparison of karyotyping and microarray-based profiling in two patients with complex karyotypes. Karyotypes and microarray-based genomic profiles from patient 4521 (panel $\mathbf{A}$ and $\mathbf{B}$ ) and 4899 (panel $\mathbf{C}$ and D). CNA observed with microarray-based genomic profiling are marked by arrows, including a $12 \mathrm{Mb}$ focal loss of chromosome 4q24q26 (blue arrow).

hematological malignancies [20,21], the application of FISH on bone marrow biopsies has been barely investigated [5-8,22] and the application of microarray-based genomic profiling not at all. This may be related to technical limitations as a poor DNA quality due to DNA degradation during FFPE fixation, decalcification and tissue processing [22,23], resulting in relatively noisy array profiles [24,25]. Recently optimized sample preparation approaches involving the fixation and decalcification process, the use of Qiagen DNA extraction kit, and improved data analysis methods, have been recommended to improve the detection of CNA by genomic arrays on FFPE samples [24]. We have optimized the fixation and decalcification procedure. Despite these adaptations, we had to set the threshold at $10 \mathrm{Mb}$ for the identification of CNA, which is much higher than required for high-molecular weight DNAs obtained from bone marrow aspirates or peripheral blood $[26,27]$. In addition the threshold for chromosome $19 \mathrm{ab}-$ normalities was increased to $30 \mathrm{Mb}$ since GC-rich regions, which are abundantly present on chromosome 19 , are less sensitive to DNA degradation (occurring in FFPE samples), leading to a more efficient PCR amplification and hence higher signal intensity. The recently introduced 
OncoScan array platform (Affymetrix) with the use of the molecular inversion probe strategy has shown to perform well with degraded DNA samples obtained from FFPE specimens [28-30].

We have evaluated the diagnostic power and limit of detection of microarray-based genomic profiling compared to FISH and Chromogenic in situ hybridizations on bone marrow biopsies. In 13 of the 15 successful in situ hybridizations concordant results were obtained by in situ hybridization and microarray-based genomic profiling (Table 1). In two other cases (4896 and 4518) in situ hybridization resulted in a false positive identified abnormality. In these two cases in situ hybridization was based on a single probe and suggested loss of chromosome segments (deletion $7 \mathrm{q}$ and loss of chromosome 8 respectively), which was not observed by microarraybased genomic profiling and karyotyping. As noted by others, the reliable detection of chromosome loss by FISH is difficult, since nuclei of cells in tissue sections are often sectioned [5]. In addition, the identification of balanced rearrangements and gain of chromosomes by FISH in tissue sections is more straight forward to detect because of a simpler probe configuration. This was confirmed in the present study were we have identified all three patients with a trisomy 8 by in situ hybridization correctly. Furthermore, the applied microarray platform exhibited a high limit of detection, i.e., CNAs present in at least $18 \%$ of the cells as determined by FISH could unambiguously be detected.

Microarray-based genomic profiling of non-fibrotic as well as fibrotic bone marrow biopsy samples demonstrated a high concordance with karyotyping on bone marrow aspirates, and discrepancies between both approaches could be explained by the complex karyotypes, the presence of marker chromosomes, the presence of balanced translocations, clonal heterogeneity or progression of the MDS. The only exception is a cytogenetically abnormal patient with a normal array profile (case 4903). The discrepancy may be explained by the sparse hematopoiesis in the biopsy, resulting in too few malignant cells present for microarray analysis, while cell culture for karyotyping, selective growth of aberrant blasts could have enabled detection of the cytogenetic aberrancies.

One important benefit of microarray-based genomic profiling as compared to FISH is its ability to detect additional chromosomal aberrations not detected by targeted probe-based assay. In four of the five patients with additional genetic abnormalities as compared to in situ hybridization, the microarray profile was indicative for a so-called complex karyotype which is associated with an adverse prognosis [2,31]. In addition a focal loss on chromosome 4q24q26 region containing the TET2 gene as demonstrated by microarray-based genomic profiling in case 4521 could not be observed by karyotyping because it was below the level of cytogenetic resolution. The protein encoded by the TET2 gene plays a key role epigenetic modification of the genome by DNA demethylation. The identification of this focal loss of the TET2 gene is of clinical relevance since it has been demonstrated that this is a recurrent genetic abnormality in MDS patients [11].

\section{Conclusions}

Our work represents the first genome wide microarray approach for the analysis for CNA in bone marrow biopsy samples in MDS patients. This is of clinical relevance for MDS patients with fibrotic bone marrows in whom cytogenetic data are lacking. In these patients microarray is able to identify cytogenetic abnormalities which are important parameters for the (revised) international prognostic scoring system for MDS patients. We demonstrate a high concordance of karyotyping on bone marrow aspirates and microarray-based genomic profiling on both non-fibrotic and fibrotic bone marrow biopsies in MDS patients. Although in situ hybridization can be applied to bone marrow biopsies, it is important to note that in situ hybridization for losses of chromosomes or chromosome segments may be misinterpreted due to technical limitations. Therefore this study demonstrates in MDS cases with failure to acquire a representative bone marrow aspirate that microarray-based genomic profiling eventually complemented with FISH for balanced prognostic relevant abnormalities (eg. 3q26/MECOM rearrangements) is a valid alternative approach to obtain information on clinical relevant cytogenetic abnormalities.

\section{Methods \\ Patients}

Twenty-six bone marrow biopsies from MDS patients with a successful cytogenetic result, were initially selected from the hospital archive. Of these 26 samples, 5 were excluded because of several reasons (as described under Results). For the remaining 19 MDS cases and 2 cases with a reactive non-fibrotic bone marrow, a goodquality microarray-based genomic profile was obtained, and these patients were further evaluated in this study. Seven MDS patients were selected because of an abnormal karyotype as determined by conventional cytogenetics on a simultaneous bone marrow aspirate (group 1). Seven other patients were selected because they had an abnormal karyotype and extensive fibrosis in the bone marrow biopsy (fibrosis grade $2 / 3$ or $3 / 3$ ) (group 2). Another 4 MDS, 1 AML and 2 reactive non-fibrotic $\mathrm{BMB}$ without copy number alterations (CNA) as determined by karyotyping were selected as controls (group 3) 
(Table 1). The diagnosis of MDS was made by combining histology, cytomorphology, clinical and cytogenetic data.

The bone marrow biopsies were fixed in Burckhardt $\mathrm{pH} 7.4$ at room temperature, followed by decalcification in EDTA 10\% pH 7.2 (room temperature) during $48 \mathrm{hrs}$. Post-decalcification tissue processing consisted of dehydration and paraffin embedding using either the Pathos Rapid Microwave Histoprocessor (Milestone, Sorisole, Italy) or the Shandon ${ }^{\text {Tx }}$ Excelsior $^{\text {Tx }}$ ES Tissue Processor (Thermo Scientific, Waltham, MA, USA).

This study was approved by the institutional review board of the Radboud university medical center and in accord with the Helsinki Declaration 1975, as revised in 2008.

\section{Karyotyping}

Hematopoietic cells from bone marrow aspirates were cultured and harvested for cytogenetic analysis by established methods. Chromosome aberrations were described according to guidelines of an International System for Human Cytogenetic Nomenclature [15].

\section{In situ hybridization}

Fluorescence in situ hybridization (FISH) was performed based on the protocol described by Gerr et al. [32]. Briefly, $4 \mu \mathrm{m}$ tissue sections were pretreated with $10 \mathrm{mM}$ sodiumcitrate pH 6.0 using a microwave, digested with $200 \mathrm{U} / \mathrm{ml}$ pepsin/ $0.01 \mathrm{M} \mathrm{HCl}$ and fixated in $1 \%$ buffered formaldehyde. The commercially available probes LSI D7S485/CEP 7 (7q31/centromere 7), LSI EGR1/D5S721/D5S23 (5q31/ 5q15.2) and LSI D20S108 (20q12) (Abbott Molecular, Des Plaines, Illinois, USA) were used according to the manufacturer's specifications. Signals were counted using an fluorescence microscope (DM4000B, Leica, Rijswijk, Netherlands). The homemade probe CEP 8 (D8Z2) labeled with biotin was detected using immunohistochemical staining (Chromogenic in situ hybridization). The immunohistochemical staining was performed by successive incubations with mouse-anti-biotine (Vector, Vector Laboratories, Burlingame, CA, USA), BrightVision Poly-HRP-Anti Ms/Rb/Rt IgG (Immunologic, Duiven, The Netherlands), and Bright-DAB (Immunologic, Duiven, The Netherlands) according to manufacturer's specifications. Interpretation was performed as described [32] and least 100 cells were evaluated per case.

\section{DNA extraction and microarray-based genomic profiling}

5-10 $\mu \mathrm{m}$ paraffin-embedded tissue sections were used to extract DNA using proteinase $\mathrm{K}$ treatment step according to the standard protocol. Subsequently, the DNA was treated with RNAase A (Qiagen, Venlo, The Netherlands) and was affinity-purified using QIAamp MinElute Columns (Qiagen, Venlo, The Netherlands). DNA sample concentration and quality were assessed by spectrophotometry and by PCR-amplification using the BIOMED-2 Control gene primer set [33]. Affinitypurified DNA samples (100 ng) that were able to amplify control gene fragments of $200 \mathrm{bp}$ or more were used for whole genome amplification (ENZO diagnostics Inc., Farmingdale, NY, USA) for subsequent microarray profiling. Microarray-based genomic profiling was carried out using a Nimblegen human CNV $3 \times 720 \mathrm{~K}$ wholegenome tilling array (Roche NimbleGen Inc., Madison, WI, USA) according to the manufacturer's protocols. Each sample was referenced against a sex-matched nontumor bone marrow biopsy sample. Data were analyzed using the NEXUS version 5 (Biodiscovery, Hawthorne, CA, USA) software package. The quality of the array hybridization was monitored by determination of the variance of difference between adjacent log ratio's after excluding the outliers (Nexus QC score). Array hybridizations with a Nexus QC above 0.4 were excluded for further analysis. Abnormal segments were identified by the FASST2 segmentation algorithm with a significance threshold of $1.0 \mathrm{E}-20$, a maximum probe spacing of $1 \mathrm{Mb}$, and a minimum number of 10 probes for a segment. The $\log 2$ thresholds were set to $0.4,0.12,-0.18$, and -0.6 to distinguish between high copy gains, single copy gains, single copy losses, and homozygous losses respectively. Only segments fulfilling the above criteria and $>10 \mathrm{Mb}$ were identified as CNA.

\section{Ethical consent}

This study is performed according to code for proper use of human tissue in the Netherlands as determined by the Federation of Medical Scientific Societies and in compliance with the Helsinki Declaration.

\section{Abbreviations}

MDS: Myelodysplastic syndrome; AML: Acute myeloid leukemia;

FISH: Fluorescence in situ hybridization; FFPE: Formalin fixed paraffin-embedded; CNA: Copy number alterations.

\section{Competing interests}

The authors declare that they have no competing interests.

\section{Authors' contributions}

MSK carried out the cytogenetic studies, participated in data analysis and drafted the manuscript. KH participated in the design of the study, collected cases, performed histological analysis of the bone marrow biopsies, carried out and analyzed in situ hybridization experiments and helped to draft the manuscript. EV developed and performed data analysis of the microarray-based genomic profiling. EK carried out microarray-based genomic profiling experiments. PvC carried out and analyzed in situ hybridization experiments. RK participated in the design of the study and helped to draft the manuscript. PG participated in the design of the study, optimized the DNA extraction procedure, participated in the data analysis and helped to draft the manuscript. All authors read and approved the final manuscript.

\section{Acknowledgements}

We gratefully acknowledge the hospitality of Prof. B. Schlegelberger and Dr. G. Goehring for hosting KH to perform part of the FISH studies at the Department of Cellular and Molecular Pathology of the Medizinische Hochschule Hannover with practical help of Markus Meyer and Willy Arndt. Arjan Buijs (UMC Utrecht) is acknowledged for sharing of cytogenetic data. 


\section{Author details}

'Department of Human Genetics, Radboud university medical center, P.O. Box 9101, 6500 HB Nijmegen, The Netherlands. ²Department of Pathology, Radboud university medical center, P.O. Box 9101, 6500 HB Nijmegen, The Netherlands

Received: 14 January 2015 Accepted: 16 April 2015

Published online: 28 May 2015

\section{References}

1. Greenberg P, Cox C, LeBeau MM, Fenaux P, Morel P, Sanz G, et al. International scoring system for evaluating prognosis in myelodysplastic syndromes. Blood. 1997;89:2079-88.

2. Greenberg PL, Tuechler H, Schanz J, Sanz G, Garcia-Manero G, Sole F, et al. Revised international prognostic scoring system for myelodysplastic syndromes. Blood. 2012;120:2454-65.

3. Fend F, Tzankov A, Bink K, Seidl S, Quintanilla-Martinez L, Kremer M, et al. Modern techniques for the diagnostic evaluation of the trephine bone marrow biopsy: methodological aspects and applications. ProgHistochemCytochem. 2008:42:203-52.

4. Humphries JE. Dry tap bone marrow aspiration: clinical significance. AmJHematol. 1990:35:247-50.

5. Miranda RN, Mark HF, Medeiros LJ. Fluorescent in situ hybridization in routinely processed bone marrow aspirate clot and core biopsy sections. Am.Pathol. 1994;145:1309-14.

6. Le Maitre CL, Byers RJ, Liu Yin JA, Hoyland JA, Freemont AJ. Dual colour FISH in paraffin wax embedded bone trephines for identification of numerical and structural chromosomal abnormalities in acute myeloid leukaemia and myelodysplasia. JClinPathol. 2001;54:730-3.

7. Korac $\mathrm{P}$, Jones M, Dominis M, Kusec R, Mason DY, Banham AH, et al. Application of the FICTION technique for the simultaneous detection of immunophenotype and chromosomal abnormalities in routinely fixed, paraffin wax embedded bone marrow trephines. JClinPathol. 2005;58:1336-8.

8. Reineke T, Jenni B, Abdou MT, Frigerio S, Zubler P, Moch H, et al. Ultrasonic decalcification offers new perspectives for rapid FISH, DNA, and RT-PCR analysis in bone marrow trephines. AmJSurgPathol. 2006;30:892-6.

9. Starczynowski DT, Vercauteren S, Telenius A, Sung S, Tohyama K, Brooks-Wilson A, et al. High-resolution whole genome tiling path array CGH analysis of CD34 + cells from patients with low-risk myelodysplastic syndromes reveals cryptic copy number alterations and predicts overall and leukemia-free survival. Blood. 2008;112:3412-24.

10. Tiu RV, Gondek LP, O'Keefe CL, Elson P, Huh J, Mohamedali A, et al. Prognostic impact of SNP array karyotyping in myelodysplastic syndromes and related myeloid malignancies. Blood. 2011;117:4552-60.

11. Langemeijer SM, Kuiper RP, Berends M, Knops R, Aslanyan MG, Massop M, et al. Acquired mutations in TET2 are common in myelodysplastic syndromes. NatGenet. 2009;41:838-42.

12. Heinrichs S, Li C, Look AT. SNP array analysis in hematologic malignancies: avoiding false discoveries. Blood. 2010;115:4157-61.

13. Thiel A, Beier M, Ingenhag D, Servan $K$, Hein M, Moeller $V$, et al. Comprehensive array CGH of normal karyotype myelodysplastic syndromes reveals hidden recurrent and individual genomic copy number alterations with prognostic relevance. Leukemia. 2011;25:387-99.

14. Fuhrmann C, Schmidt-Kittler O, Stoecklein NH, Petat-Dutter K, Vay C, Bockler $\mathrm{K}$, et al. High-resolution array comparative genomic hybridization of single micrometastatic tumor cells. Nucleic Acids Res. 2008:36:e39.

15. Shaffer LG, McGowan-Jordan J, Schmid M: ISCN 2013: An International System for Human Cytogenetic Nomenclature. S. Karger; 2013

16. Simons A, Sikkema-Raddatz B, de Leeuw N, Konrad NC, Hastings RJ, Schoumans J. Genome-wide arrays in routine diagnostics of hematological malignancies. Hum Mutat. 2012;33:941-8. doi:10.1002/humu.22057.

17. Bajaj R, Xu F, Xiang B, Wilcox K, Diadamo AJ, Kumar R, et al. Evidence-based genomic diagnosis characterized chromosomal and cryptic imbalances in 30 elderly patients with myelodysplastic syndrome and acute myeloid leukemia. MolCytogenet. 2011:4:3

18. Arenillas L, Mallo M, Ramos F, Guinta K, Barragan E, Lumbreras E, et al. Single nucleotide polymorphism array karyotyping: a diagnostic and prognostic tool in myelodysplastic syndromes with unsuccessful conventional cytogenetic testing. Genes Chromosomes Cancer. 2013;52:1167-77. doi:10.1002/gcc.22112.
19. Makishima H, Rataul M, Gondek LP, Huh J, Cook JR, Theil KS, et al. FISH and SNP-A karyotyping in myelodysplastic syndromes: improving cytogenetic detection of del (5q), monosomy 7, del (7q), trisomy 8 and del (20q). Leuk Res. 2010;34:447-53. doi:10.1016/j.leukres.2009.08.023.

20. Verburgh E, Achten R, Maes B, Hagemeijer A, Boogaerts M, De Wolf-Peeters

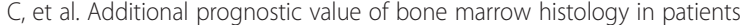
subclassified according to the International Prognostic Scoring System for myelodysplastic syndromes. JClinOncol. 2003;21:273-82.

21. Tricot G, Vlietinck R, Boogaerts MA, Hendrickx B, De Wolf-Peeters C, Van den Berghe $\mathrm{H}$, et al. Prognostic factors in the myelodysplastic syndromes: importance of initial data on peripheral blood counts, bone marrow cytology, trephine biopsy and chromosomal analysis. BrJHaematol. 1985;60:19-32.

22. Neat MJ, Moonim MT, Dunn RG, Geoghegan H, Foot NJ. Fluorescence in situ hybridisation analysis of bone marrow trephine biopsy specimens; an additional tool in the diagnostic armoury. J Clin Pathol. 2013;66:54-7. doi:10.1136/jclinpath-2012-201131.

23. Groenen PJ, Blokx WA, Diepenbroek C, Burgers L, Visinoni F, Wesseling $P$, et al. Preparing pathology for personalized medicine: possibilities for improvement of the pre-analytical phase. Histopathology. 2011;59:1-7. doi:10.1111/j.1365-2559.2010.03711.x.

24. Tuefferd M, De BA, Van DW I, Talloen W, Verbeke T, Carvalho B, et al. Genome-wide copy number alterations detection in fresh frozen and matched FFPE samples using SNP 6.0 arrays. Genes ChromosomesCancer. 2008:47:957-64

25. Costantini M, Clay O, Auletta F, Bernardi G. An isochore map of human chromosomes. Genome Res. 2006;16:536-41. doi:10.1101/gr.4910606.

26. Simons A, Stevens-Kroef M, El Idrissi-Zaynoun N, van Gessel S, Olde Weghuis D, van den Berg E, et al. Microarray-based genomic profiling as a diagnostic tool in acute lymphoblastic leukemia. Genes ChromosomesCancer. 2011;50:969-81.

27. Stevens-Kroef MJ, van den Berg E, Olde Weghuis D, Geurts van Kessel A, Pfundt R, Linssen-Wiersma $M$, et al. Identification of prognostic relevant chromosomal abnormalities in chronic lymphocytic leukemia using microarray-based genomic profiling. Mol Cytogenet. 2014;7:3. doi:10.1186/ 1755-8166-7-3

28. Schiffman JD, Lorimer PD, Rodic V, Jahromi MS, Downie JM, Bayerl MG, et al. Genome wide copy number analysis of paediatric Burkitt lymphoma using formalin-fixed tissues reveals a subset with gain of chromosome $13 q$ and corresponding miRNA over expression. Br J Haematol. 2011;155:477-86. doi:10.1111/j.1365-2141.2011.08883.x.

29. Wang L, Lawrence MS, Wan Y, Stojanov P, Sougnez C, Stevenson K, et al. SF3B1 and other novel cancer genes in chronic lymphocytic leukemia. N Engl J Med. 2011;365:2497-506. doi:10.1056/NEJMoa1109016.

30. van Engen-van Grunsven AC, Baar MP, Pfundt R, Rijntjes J, Küsters-Vandevelde $H V$, Delbecq A-L, Keunen JE, Klevering JB, Wesseling P, Blokx WA, Groenen PJ. Whole genome copy number analysis identifies new leads for chromosomal aberrations involved in the oncogenesis and metastastic behavior of uveal melanomas. Melanoma Research 2015; ahead of print. doi:10.1097/CMR.0000000000000152.

31. Grimwade D, Hills RK, Moorman AV, Walker H, Chatters S, Goldstone AH, et al. National cancer research institute adult leukaemia working G. Refinement of cytogenetic classification in acute myeloid leukemia: determination of prognostic significance of rare recurring chromosomal abnormalities among 5876 younger adult patients treated in the united kingdom medical research council trials. Blood. 2010;116:354-65. doi:10.1182/blood-2009-11254441.

32. Gerr H, Gadzicki D, Kreipe H, Schlegelberger B, Wilkens L. Fluorescence in situ hybridization reveals closely correlated results in cytological and histological specimens of hematological neoplasias compared to conventional cytogenetics. Pathobiology. 2006;73:271-9.

33. van Dongen JJ, Langerak AW, Bruggemann M, Evans PA, Hummel M, Lavender FL, et al. Design and standardization of PCR primers and protocols for detection of clonal immunoglobulin and T-cell receptor gene recombinations in suspect lymphoproliferations: report of the BIOMED-2 Concerted Action BMH4-CT98-3936. Leukemia. 2003;17:2257-317. doi:10.1038/sj.leu.2403202. 